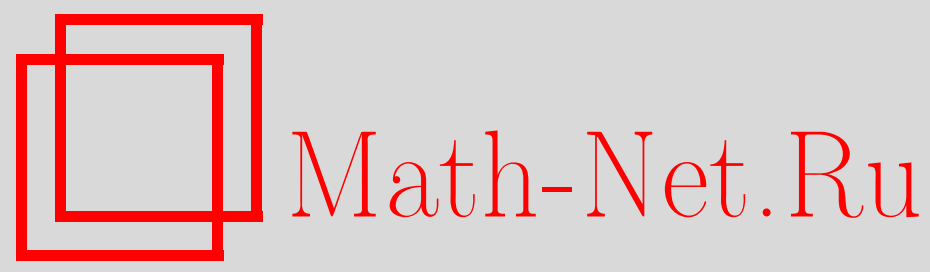

С. Я. Серовайский, Арифметические распределения и секвенциальное продолжение бинарных отношений, Матем. заметки, 1999, том 65, выпуск 6, 836-853

DOI: https://doi.org/10.4213/mzm1120

Использование Общероссийского математического портала Math-Net.Ru подразумевает, что вы прочитали и согласны с пользовательским соглашением http://www . mathnet.ru/rus/agreement

Параметры загрузки:

IP: 3.93 .64 .190

26 апреля 2023 г., $17: 12: 09$

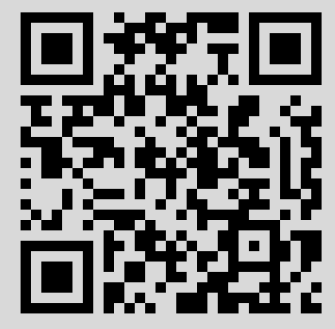




\title{
АРИФМЕТИЧЕСКИЕ РАСПРЕДЕЛЕНИЯ И СЕКВЕНЦИАЛЬНОЕ ПРОДОЛЖЕНИЕ БИНАРНЫХ ОТНОШЕНИЙ
}

\author{
С. Я. Серовайский
}

\begin{abstract}
С помощью некоторой итерационной процедуры определяется достаточно шшрокий класс арифметических распределений, на котором определены и непрерывны операции сложения, умножения и дифференцирования. Проводится согласование введенного умножения арифометических распределений с известньми методами определения произведения обобщенных функций. Установленные результаты могут быть использованы для обоснования предельных переходов при исследовании нелинейных задач математической физики.

На основе формализации применяемой схемы описывается процедура построения некоторого продолжения бинарных отношений, названного секвенциальным продолжением, с плотного подмножества на все топологическое пространство. Эти результаты распространяются на операции первого порядка. Показьвается, что секвенциальное продолжение операции дифференцирования с множества бесконечно дифференцируемых функций на пространство распределений с точностью до изоморфизма совпадает с обобщенным дифференцированием распределений.
\end{abstract}

Библиография: 26 названий.

Задачи о продолжении тех или иных структур возникают в различных разделах математики и имеют многочисленные приложения (см., например, [1]-[3]). Эта проблема имеет чрезвычайно важное значение в теории распределений, где не определена естественная операция умножения. С необходимостью перемножения объектов, названных впоследствии обобщенными функциями, впервые столкнулся П. Дирак при исследовании нелинейных задач квантовой электродинамики [4]. Чувствуя возникающие при этом принципиальные трудности, создатель фундамента теории обобщенных функций Л. Шварц отмечал, что аппарат распределений приспособлен исключительно для систем линейной природы [5]. Грандиозные успехи, достигнутые математической и теоретической физикой за счет использования теории обобщенных функций [5]-[8], и отсутствие естественного определения произведения распределений, а значит, принципиальные осложнения при интерпретации обобщенных решений нелинейных уравнений стали мощньгм стимулом развития теории умножения распределений.

Начиная с работ Я. Микусинского [8], эта проблема решается на основе секвенциального подхода. При этом каждое распределение представляется классом регулярных функций. Тогда умножение обобщенных функций может быть интерпретировано 
как объект, соответствуюший классу произведений регулярных функций, представляюших рассматриваемые распределения. При этом семейство представляющих функций обычно выбирается таким образом, чтобы оно обладало структурой алгебры. На нем задается некоторое отношение эквивалентности, характеризуюшее соответствующий идеал. В качестве класса объектов, интерпретируемых как обобщенные распределения, и выбирается получаемая при этом факторалгебра. Тем самьм, замыкание множества распределений относительно операции умножения получается в процессе ее продолжения с множества представляющих регулярных функций. Среди исследований этого направления, различающихся лишь выбором соответствующего класса представлений и эквивалентности на нем, отметим работы Г. Тильмана [9], Г. Бремермана [10], В.К. Иванова [11], К. Келлера [12], Л. Берга [13], В.П. Маслова [14], Ж.П. Коломбо [15], [16], Ю. В. Егорова [17], В.М. Шелковича [18] и др. Получаемые в результате достаточно широкие и содержательные семейства обобщенных функций с успехом используются при интерпретации обобщенньх решений широкого класса нелинейных задач математической физики [14], [16]-[19]. Однако, теория умножения распределений еше далека от завершения. Так, Егоров в своем обзоре [17] отмечает, что "несмотря на отдельные успехи, связанные с определением произведения для распределений из специальных классов, в общем случае эта задача неразрешима".

Используемьй способ расширения множества обобщенных функций является достаточно эффективным. Однако его несомненная простота и естественность с алгебраической точки зрения предопределили трудности топологической природы. В указанных работах, как правило, не определяется топология на расширенном множестве распределений, а если таковая и вводится, то без согласования с алгебраической структурой множества [17]. Это обстоятельство не позволяет воспользоваться получаемыми результатами для решения топологических задач, возникающих в теории нелинейных уравнений математической физики.

Наряду с достаточно успешно решаемыми на основе подобных результатов вопросами интерпретации их решений несомненный интерес представляет также проблема перехода к пределу в нелинейных членах при обосновании качественных и количественных методов исследования этих уравнений. В линейном случае наличие простейших априорных оценок позволяет без труда перейти к пределу и добиться желаемого результата. В нелинейных уравнениях проблема обоснования предельного перехода приводит к существенным и подчас непреодолимьм трудностям, поскольку из слабой сходимости функций, вообще говоря, не следует сходимость соответствуюших нелинейных выражений от них, в частности, их произведений. В ряде случаев желаемый результат удается получить с помощью специального аппарата - методов монотонности, компактности и т.п. (см., например, [20], [21]). Однако, для этого требуются либо весьма специфические свойства рассматриваемого оператора (метод монотонности), либо достаточно сильные априорные оценки (метод компактности), что далеко не всегда удается реализовать на практике.

Обоснование предельных переходов было бы естественно проводить на расширенном множестве распределений при условии, что на нем определена содержательная топология, согласованная с его алгебраической структурой. Действительно, имея какие-либо априорные оценки, мы получаем слабую сходимость последовательностей в соответствуюших функциональных классах, а значит (как правило), и в топологии простран- 
ства классических распределений. Если последнее непрерьвно вложено в расширенное пространство распределений, на котором определена непрерывная операция умножения, то в результате получается сходимость произведений элементов рассматриваемых последовательностей. Учитьвая, что в большинстве встречаюшихся на практике задач математической физики нелинейные члены характеризуются как раз произведениями неизвестных функций и их производных, обоснование предельных переходов в таких задачах можно было бы осуществить указанным способом. Таким образом, определение именно непрерывного умножения распределений представляется весьма актуальной задачей.

В настоящей работе предлагается итерационная процедура расширения множества распределений, на каждом шаге которой параллельно вводится алгебраическая и топологическая структура, что позволяет добиться их согласованности. В результате получается достаточно широкий класс арифметических распределений, на котором определены и непрерывны операции сложения, умножения и дифференцирования, вследствие чего установленные результаты могут быть использованы для анализа широкого класса нелинейных задач математической физики. Отдельные результаты в этом направлении были получены в работах автора [22], [23]. Показывается, что описанная техника может использоваться для построения продолжения бинарных отношений с плотного подмножества на все топологическое пространство.

1. Арифметические распределения. Рассмотрим множество $D(\Omega)$ бесконечно дифференцируемых финитных функций в области $\Omega$ с топологией индуктивного предела и сопряженное к нему пространство распределений $D^{\prime}(\Omega)$. Учитьвая плотность вложения пространства $D(\Omega)$ в $D^{\prime}(\Omega)$, установим, что для любого распределения сушествует сходящаяся к нему в топологии $D^{\prime}(\Omega)$ последовательность бесконечно дифференцируемых функций. Обозначим через $\Psi$ множество всех сходящихся в смысле $D^{\prime}(\Omega)$ последовательностей бесконечно дифференцируемых функций. Определим на нем отношение эквивалентности $\psi$, считая условие $\left\{x_{k}\right\} \psi\left\{y_{k}\right\}$ выполненным, если соответствующие пределы совпадают. Рассмотрим фактормножество $D_{p}=\Psi / \psi$, элементы которого будем называть простыми распределениями в области $\Omega$.

Для любого $x \in D^{\prime}(\Omega)$ определим класс эквивалентности $I x=\left[\left\{x_{k}\right\}\right]_{\psi}$ по отношению $\psi$ с представителем $\left\{x_{k}\right\}$ - последовательностью элементов из $D(\Omega)$, сходящейся к $x$. Значение $I x$ принадлежит множеству $D_{p}$ и не зависит от выбора сходящейся к $x$ последовательности. Тем самым, задан оператор $I: D^{\prime}(\Omega) \rightarrow D_{p}$, который оказьвается биекцией, причем соответствующий обратный оператор характеризуется соотношениeM

$$
I^{-1} x=\lim x_{k} \quad \forall\left\{x_{k}\right\} \in X .
$$

Оператор $I$ будем назьвать секвенииальным изоморфизмом пространства $D^{\prime}(\Omega)$ относительно множества $D(\Omega)$. Положим $Y_{x}=I(D(\Omega))$.

Для произвольного натурального числа $m$ зададим класс последовательностей

$$
\Pi_{m}=\left\{\left\{x_{k}\right\} \subset D(\Omega) \mid \exists\left\{x_{k}^{i}\right\} \in \Psi, i=1, \ldots, m: x_{k}=\prod_{i=1}^{n} x_{k}^{i} \quad \forall k\right\} .
$$

Очевидно, $\Pi_{1}=\Psi$, однако при $m>1 \Psi$ является собственным подмножеством $\Pi_{m}$. Действительно, пусть $\left\{d_{k}\right\}$ есть некоторая последовательность из $D(\Omega)$, сходящаяся в 
топологии $D^{\prime}(\Omega)$ к $\delta$-функции $\delta$, сосредоточенной в некоторой точке области $\Omega$. Тогда последовательность квадратов $\left\{\left(d_{k}\right)^{2}\right\}$ не сходится в пространстве $D^{\prime}(\Omega)$ (это и является проявлением неопределенности естественного квадрата от $\delta$-функции [8]), а значит, не является элементом множества $\Psi$. Однако, $\left(d_{k}\right)^{2}$ представляет собой произведение двух (совпадающих) элементов, каждьй из которых образует сходящуюся на $D^{\prime}(\Omega)$ последовательность. Таким образом, справедливо включение $\left\{\left(d_{k}\right)^{2}\right\} \in \Pi_{2}$.

Введем на $\Pi_{m}$ эквивалентность $\pi_{m}$, считая условие $\left\{x_{k}\right\} \pi_{m}\left\{y_{k}\right\}$ выполненным, если существуют последовательности $\left\{x_{k}^{i}\right\},\left\{y_{k}^{i}\right\}$ из $\Psi$ такие, что справедливы соотношения

$$
x_{k}=\prod_{i=1}^{m} x_{k}^{i}, \quad y_{k}=\prod_{i=1}^{m} y_{k}^{i} \quad \forall k: \quad\left\{x_{k}^{i}\right\} \psi\left\{y_{k}^{i}\right\}, \quad i=1, \ldots, m .
$$

Обозначим через П объединение семейства $\left\{\Pi_{m}\right\}$. Определим $X_{m}=\Pi_{m} / \pi_{m}$. Mножеством $D^{n}$ натуральны распределений на $\Omega$ назовем объединение семейства $\left\{X_{m}\right\}$.

Для любого $x \in D^{n}$ существует номер $m$ такой, что $x \in X_{m}$. Тогда найдется последовательность $\left\{x_{k}\right\} \in \Pi_{m}$ такая, что справедливо равенство $x=\left[\left\{x_{k}\right\}\right]_{m}$, правая часть которого представляет собой класс эквивалентности по отношению $\pi_{m}$ с представителем $\left\{x_{k}\right\}$. Множество $D^{n}$ сушественно шире, чем $D_{p}$. Так, $\delta$-функцию с точностью до изоморфизма можно считать простым распределением. В то же время в классе $D^{\prime}(\Omega)$ (а значит, и в $D_{p}$ ) не имеет смысла степень от $\delta$-функции. Однако, под $\delta^{n}$ можно понимать класс эквивалентности $\left[\left(d_{k}\right)^{n}\right]_{n}$, т.е. натуральное распределение.

Определим оператор $A_{m, n}: X_{m} \times X_{n} \rightarrow X_{m+n}$ с помошью равенства

$$
A_{m, n}(x, y)=\left[\left\{x_{k}, y_{k}\right\}\right]_{m+n} \quad \forall\left\{x_{k}\right\} \in x \in X_{m},\left\{y_{k}\right\} \in y \in X_{n} .
$$

Задавая на множестве $D^{n}$ умножение следующим образом:

$$
x \cdot y=A_{m, n}(x, y) \quad \forall x \in X_{m}, y \in X_{n},
$$

заключаем, что произведение любых натуральных распределений является элементом из $D^{n}$. Каждое натуральное распределение представимо в виде произведения простых распределений подобно тому, как любое натуральное число разлагается в произведение простых чисел, чем и объясняется принятая терминология. Так, элемент $\delta^{n}$ действительно является $n$-кратным произведением на себя простого распределения $\delta$. Однако, в отличие от чисел здесь естественно нарушаются утверждения основной теоремы арифометики, т.е. подобное разложение не единственно.

На множестве натуральных распределений не определена сумма элементов. Замыкая его относительно операции сложения, приходим к более широкому классу объектов. Для любого номера $n$ рассмотрим семейство

$$
\Sigma_{n}=\left\{\left\{x_{k}\right\} \subset D(\Omega) \mid \exists\left\{x_{k}^{i}\right\} \in \Pi, i=1, \ldots, n: \quad x_{k}=\sum_{j=1}^{n} x_{k}^{j} \forall k\right\} .
$$

Очевидно, $\Sigma_{1}=\Pi$, однако при $n>1 \Pi$ является собственным $\Sigma_{n}$. В частности, для полинома

$$
p_{n}(t)=\sum_{j=0}^{n} a_{j} t^{j}, \quad a_{j} \in \mathbb{R}, \quad j=0, \ldots, r
$$


последовательность $\left\{p_{n}\left(d_{k}\right)\right\}$ принадлежит классу $\Sigma_{n+1}$, но не П.

Элементы $\left\{x_{k}\right\},\left\{y_{k}\right\}$ на $\Sigma_{n}$ будем считать әквивалентными в смысле отношения $\sigma_{n}$, если существуют номера $m_{j}$ и последовательности $\left\{x_{k}^{j}\right\},\left\{y_{k}^{j}\right\}$ из $\Pi_{m_{j}}, j=1, \ldots, n$, такие, что справедливы соотношения

$$
x_{k}=\sum_{j=1}^{n} x_{k}^{j}, \quad y_{k}=\sum_{j=1}^{n} y_{k}^{j} \quad \forall k: \quad\left\{x_{k}^{j}\right\} \pi_{m_{j}}\left\{y_{k}^{j}\right\} \forall j .
$$

Введем фактормножество $Y_{n}=\Sigma_{n} / \sigma_{n}$. Множеством $D^{i}$ иельх распределенийна $\Omega$ назовем объединение семейства $\left\{Y_{n}\right\}$. Элементы множеств $D_{p}, D^{n}$ и $D^{i}$ будем называть также арифметическими распределениями. Подобные классы были определены в [22], [23] на основе более громоздкой схемы и без анализа их структуры.

Для любого $x \in D^{i}$ сушествует номер $n$ такой, что $x \in Y_{n}$, а значит, найдется последовательность $\left\{x_{k}\right\}$ из семейства $\Sigma_{n}$ такая, что справедливо равенство $x=\left[\left\{x_{k}\right\}\right]^{n}$, в правой части которого находится класс эквивалентности по отношению $\sigma_{n}$ с представителем $\left\{x_{k}\right\}$. Нетривиальным примером целого распределения служит объект

$$
p_{n}(\delta)=\left[\left\{p_{n}\left(d_{k}\right)\right\}\right]^{n+1} .
$$

Зададим операторы $B_{m, n}: Y_{m} \times Y_{n} \rightarrow Y_{m+n}, C_{m, n}: Y_{m} \times Y_{n} \rightarrow Y_{m n}$ :

$$
B_{m, n}(x, y)=\left[\left\{x_{k}+y_{k}\right\}\right]^{m+n}, \quad C_{m, n}(x, y)=\left[\left\{x_{k} y_{k}\right\}\right]^{m n} .
$$

Определим сложение и умножение в классе целых распределений:

$$
x+y=B_{m, n}(x, y), \quad x \cdot y=C_{m, n}(x, y) \quad \forall x \in Y_{m}, y \in Y_{n} .
$$

Множество $D^{i}$ замкнуто относительно этих операций. Любое целое распределение разлагается в сумму натуральных распределений. В частности, справедливо равенство

$$
p_{n}(\delta)=\sum_{j=0}^{n} a_{j} \delta^{j}
$$

т.е. мы действительно имеем дело с полиномом от $\delta$-функции.

Если $\partial$ - оператор дифференширования (произвольного порядка) на $D^{\prime}(\Omega)$ по какому-либо аргументу, то дифференцирование на $D_{p}$ характеризуется соотношением

$$
\partial x=I\left(\partial I^{-1} x\right) \quad \forall x \in D_{p} .
$$

Любое натуральное распределение $x$ представимо в виде произведения некоторого числа простых распределений $x^{1}, \ldots, x^{m}$. Тогда его производная определяется по формуле

$$
\partial x=\sum_{i=1}^{n} \prod_{\substack{j=1 \\ j \neq i}} x^{i} \cdot \partial x^{j}
$$

и является целым распределением. Произвольное целое распределение является суммой натуральных распределений. Его производную можно определить как сумму производных соответствующих натуральных распределений. Таким образом, дифференцирование оказьвается линейным оператором на множестве $D^{i}$. Определив естественным образом умножение на скаляр на множестве целых распределений, приходим к следующему утверждению. 
Теорема 1. Множество иелых распределений $D^{i}$ обладает структурой дифференциальной коммутативно-ассочиативной алгебры.

2. Арифметические распределения. Согласованный вариант. $\mathrm{K}$ настоящему времени существует значительное количество способов определения умножения и алгебры на множестве распределений (см., например, [8]-[18]), что само по себе является лучшим свидетельством отсутствия достаточно удовлетворительной теории изучаемых явлений. На принципиальные трудности, возникаюшие в процессе перемножения распределений, обратил внимание еще Шварц, которьй показал, что на достаточно широком множестве обобшенных функций умножение, согласующееся с естественным умножением функций, не может быть ассоциативным [24]. Отсюда следует, что при определении произведения обобщенных функций неминуемо придется отказаться от того или иного "естественного" свойства произведения. В связи с этим имеются существенные различия в определении умножения распределений. В ряде случаев принципиально отказываются от свойств ассоциативности [8]-[10], [15]-[17]. Ассоциативность может быть достигнута либо при отсутствии согласования с умножением на мультипликаторы [10], либо с потерей коммутативности [12], [13], либо за счет отказа от однозначности произведения [10], [11] (однозначность умножения отсутствует также и в [17]), либо при рассмотрении не всех распределений, а лишь некоторых классов [18].

Введенное вьше умножение распределений обладает рядом замечательных свойств: определено на всем множестве обобщенных функций (и даже на существенно более широком классе целых распределений), однозначно, коммутативно и ассоциативно. Однако, наличие последнего свойства в соответствии с тезисом Шварца неминуемо приводит к несогласованности (в ряде случаев) с естественным умножением функций, а также с умножениями типа Микусинского [8], для которых подобное согласование имеется (см. также [10], [15]-[17]). Покажем, что при незначительной модификации приведенных выше результатов можно добиться указанного согласования, что естественно сопровождается отказом от ассоциативности.

В описанной выше схеме произведение двух простых (а с точностью до изоморфизма - и классических) распределений заведомо является натуральным, но не простым, подобно тому, как произведение простых чисел никогда не дает простое число, а принадлежит более широкому классу натуральных чисел. Микусинский предложил достаточно естественный (согласуюшийся с обычным умножением) способ определения произведения распределений, который в некоторых случаях давал обычную обобщенную функцию [8]. В частности, если $\left\{x_{k}\right\},\left\{y_{k}\right\}$ - последовательности бесконечно дифференцируемых функций, сходяшиеся в топологии $D^{\prime}(\Omega)$ к некоторым распределениям $x$ и $y$ соответственно, и существует предел (в той же топологии) последовательности произведений $\left\{x_{k} y_{k}\right\}$, то этот предел можно считать результатом произведения рассматриваемых распределений. Отталкиваясь от этой идеи Микусинского, подпроизведением двух распределений понимают некоторый объект, определяемьй как произведение соответствуюших представлений и, вообще говоря, не являющийся классической обобщенной функцией, но в виде исключения (в частности, когда произведение Микусинского имеет смысл) оказываюшийся таковой. При этом разные авторы отдавали предпочтение какому-либо своему типу представления. Так, Микусинский рассматривал свертки с $\delta$-функциями [8], Г. Бремерман и Л. Дюран [10], Г. Тильман [9], В. К. Иванов [11] - ана- 
литические представления Коши, Л. Берг [13] - аналитические представления Пуассона, В.П. Маслов [14] и В. М. Шелкович [18] - асимптотические разложения, Ж. П. Коломбо - аналитические функционалы с умеренньм ростом производных [15], [16], [19].

Проведем согласование множества арифметических распределений и умножения на нем с известньми результатами. Для любых простых распределений $x, y$ определим $x \cdot y=I\left(I^{-1} x \cdot I^{-1} y\right)$, если произведение классических распределений в скобках, понимаемое, например, в смысле Микусинского, существует. При этом будем говорить, что выполнено отношение $x \mu y$, в противном случае - что справедливо отношение $x \nu y$.

Для всех $x, y \in X_{1}$ сушествуют последовательности $\left\{x_{k}\right\},\left\{y_{k}\right\}$ из $\Psi$ такие, что $x=$ $\left[\left\{x_{k}\right\}\right]_{1}, y=\left[\left\{y_{k}\right\}\right]_{1}$. Определим в качестве $A_{11}(x, y)$ величину $\left[\left\{x_{k} y_{k}\right\}\right]_{1}$ при условии $x \mu y$ и $\left[\left\{x_{k} y_{k}\right\}\right]_{2}$ в противном случае. Обозначая через $X_{2}$ множество всех значений $A_{11}(x, y)$ при $x, y \in X_{1}$, получаем оператор $A_{11}: X_{1} \times X_{1} \rightarrow X_{2}$ такой, что $A_{11}(x, y)=$ $A_{11}(y, x)$. Для любого $x \in X_{2}$ справедливо вложение $x \subset \Pi_{2}$.

Для любых $x \in X_{1}, y \in X_{2}$ существуют простые распределения $y_{1}, y_{2}$ и последовательности $\left\{x_{k}\right\},\left\{y_{k}^{i}\right\}$ из $\Psi$ такие, что $y=A_{11}\left(y_{1}, y_{2}\right), x=\left[\left\{\xi_{k}\right\}\right]_{1}, y_{i}=\left[\left\{y_{k}^{i}\right\}\right]_{1}$, $i=1,2$. При $y_{1} \mu y_{2}$ в качестве $A_{12}(x, y)$ выберем значение $A_{11}(x, y)$. В случае $y_{1} \nu y_{2}$ под $A_{12}(x, y)$ будем понимать величину $A_{11}\left(A_{11}\left(x, y_{i}\right), y_{j}\right)$ при $x \mu y_{i}, x \nu y_{j}$ для $i \neq j ;$ класс $\left[\left\{x_{k} y_{k}^{1} y_{k}^{2}\right\}\right]_{3}$ при $x \nu y_{j}, j=1,2$, и объединение $A_{11}\left(A_{11}\left(x, y_{1}\right), y_{2}\right) \cup A_{11}\left(A_{11}\left(x, y_{2}\right), y_{1}\right)$ при $x \mu y_{i}, i=1,2$. Обозначая через $X_{3}$ множество всех $A_{12}(x, y)$ при $x \in X_{1}, y \in X_{2}$, получаем отображение $A_{12}: X_{1} \times X_{2} \rightarrow X_{3}$. Оператор $A_{21}: X_{2} \times X_{1} \rightarrow X_{3}$ характеризуется равенством $A_{21}(y, x)=A_{12}(x, y)$. Для любого $x \in X_{3}$ справедливо вложение $x \subset \Pi_{3}$.

При $x \in X_{1}, y \in X_{3}$ найдутся величины $y_{1} \in X_{1}, y_{2} \in X_{2}$ такие, что $y=A_{12}\left(y_{1}, y_{2}\right)$. В качестве $A_{13}(x, y)$ выберем значение $A_{11}(x, y)$ при $y \in X_{1}$ и $A_{12}(x, y)$ при $y \in X_{2} \backslash X_{1}$. В случае $y \in X_{3} \backslash X_{2}$ под $A_{13}(x, y)$ будем понимать величину $A_{12}\left(A_{11}\left(x, y_{1}\right), y_{2}\right)$ при $A_{11}\left(x, y_{1}\right) \in X_{1}$ и $A_{12}\left(x, y_{2}\right) \notin X_{2} ; A_{12}\left(y_{1}, A_{12}\left(x, y_{2}\right)\right)$, если оба последних соотношения не выполняются; объединение этих значений, если $A_{11}\left(x, y_{1}\right) \in X_{1}$ и $A_{12}\left(x, y_{2}\right) \in X_{2}$; класс $\left[\left\{x_{k} y_{k}\right\}\right]_{4}$, если два последних включения не имеют места, где $x=\left[\left\{x_{k}\right\}\right]_{1}, y=$ $\left[\left\{y_{k}\right\}\right]_{3}$. Обозначая через $X_{4}^{13}$ совокупность всех $A_{13}(x, y)$, получаем оператор $A_{13}$ : $X_{1} \times X_{3} \rightarrow X_{4}^{13}$. Аналогично вводятся множество $X_{4}^{31}=X_{4}^{13}$ и отображение $A_{31}$ : $X_{3} \times X_{1} \rightarrow X_{4}^{31}$. Пользуясь описанной процедурой, определим множество $X_{4}^{22}$ и оператор $A_{22}: X_{2} \times X_{2} \rightarrow X_{4}^{22}$. В качестве $X_{4}$ выбираем объединение $X_{4}^{22}$ и $X_{4}^{13}$. Любой его элемент является подмножеством $\Pi_{4}$.

На шаге $r$ при $r>3$ определяются множества $X_{r}^{m n}$ с $r=m+n$ и операторы $A_{m n}: X_{m} \times X_{n} \rightarrow X_{r}^{m n}$. Под $X_{r}$ понимается объединение семейства $\left\{X_{r}^{m n}\right\}$ по соответствуюшим значениям $m$ и $n$. Любой элемент $X_{r}$ является подмножеством $\Pi_{r}$. Объединение семейства $\left\{X_{r}\right\}$ будем обозначать через $D_{n}$ и вновь назьвать множсеством натуральных распределений.

Обозначим через $J_{m}$ вложение множества $X_{m}$ в $D_{n}$. Тогда оператор $K=J_{1} I$ будет вложением из $D^{\prime}(\Omega)$ в $D_{n}$. Для любых натуральных распределений $x, y$ найдутся номера $m, n$ и $x^{\prime} \in X_{m}, y^{\prime} \in X_{n}$ такие, что справедливы равенства $x=J_{m} x^{\prime}, y=J_{n} y^{\prime}$. Определим умножение

$$
x \cdot y=J_{m+n} A_{m n}\left(x^{\prime}, y^{\prime}\right)
$$

Очевидно, множество натуральных распределений замкнуто относительно умноже- 
ния. При этом если существует произведение классических распределений по Микусинскому, то оно с точностью до изоморфизма совпадает с произведением соответствуюших простых распределений. Если воспользоваться аналитическим представлением Коши распределений, а вместо последовательностей рассматривать направленности, сходящиеся в смысле Мура-Смита в топологии $D^{\prime}(\Omega)$ (вместо этого можно воспользоваться теорией фильтров), то введенное умножение сводится (с точностью до изоморфизма) к умножению Бремермана-Дюрана [10], а класс простых распределений будет соответствовать гиперраспределениям Иванова [11]. Выбирая другие типы представлений обобщенных функций и соответствуюшие им способы перемножения (например, в смысле Коломбо [15], [16], [19]), можно согласовать с ними умножение получаемых в результате классов натуральных распределений. Неминуемым следствием подобного согласования оказьвается следующий результат.

Теорема 2. Умножение на $D_{n}$ неассочиативно.

ДокАЗАТЕЛЬСтво. В соответствии с примером Шварца [24] определим распределения $\xi=\xi(t)=1 / t, \eta=\eta(t)=t, \zeta=\zeta(t)=\delta(t)$ есть $\delta$-функция, сосредоточенная в нуле. Обозначая $x=K \xi, y=K \eta, z=K \zeta$, имеем

$$
\begin{aligned}
& (x \cdot y) \cdot z=K(\xi \cdot \eta) \cdot z=K e \cdot K \zeta=K(e \cdot \delta)=K \delta=z, \\
& x \cdot(y \cdot z)=x \cdot K(\eta, \zeta)=K\left(\frac{1}{t}\right) \cdot K o=K\left(\frac{1}{t} \cdot o\right)=K o=o,
\end{aligned}
$$

где через о и е обозначены функции, тождественно равные 0 и 1 . Теорема доказана.

Повторяя описанные вьше рассуждения с заменой множества $D^{n}$ на $D_{n}$, приходим к новому классу иелых распределений $D_{i}$, на котором определены сложение и неассоциативное умножение, согласующееся с тем или иным известным способом перемножения распределений, а также дифференцирование произвольного порядка по любому аргументу. Тем самьм, любой элемент класса $D_{i}$ с точностью до изоморфизма совпадает с некоторым объектом из соответствующего расширения множества распределений (например, с обобщенной функцией Коломбо). При этом справедливо следующее утверждение.

ТЕорема 3. Множество иелых распределений $D_{i}$ обладает структурой дифференииальной коммутативной неассочиативной алгебры.

3. Топология арифметических распределений. Выше были определены классы арифметических распределений, в которых умножение либо ассоциативно, но не согласуется с известными результатами в этой области (множество $D^{i}$ ), либо обладает свойством согласованности, но в отсутствии ассоциативности (множество $D_{i}$ ). При практическом использовании первого класса объектов может возникнуть ощущение, что мы имеем дело с неестественной операцией умножения, хотя, как отмечалось ранее, подобными свойствами обладают и некоторые другие схемы перемножения распределений, а в ряде случаев считается оправданным даже отсутствие однозначности произведения. Во втором случае подобные сомнения отпадают, а к неассоциативности умножения обобщенных функций со времен Микусинского принято относиться достаточно спокойно. Однако, возникает естественньй вопрос: зачем нужны предлагаемые вьше 
конструкции, коль скоро аналогичные результаты были установлены ранее Коломбо, Егоровым и другими авторами, причем более простым способом?

Достаточно естественньй с алгебраической точки зрения способ расширения множества обобщенных функций (определение алгебры представлений, выбор идеала на ней, переход к соответствующей факторалгебре) оказывается слишком "быстрым" и не позволяет ввести на получаемом расширении содержательную топологию, согласованную с алгебраическими операциями. В известных работах топология на соответствуюших расширениях, как правило, вообе не вводится, а если и вводится, то без согласования с алгебраической структурой множества. В связи с этим вполне естественным представляется утверждение Егорова о том, что подобная топология "до сих пор не использовалась практически" [17]. Описанньй выше более "медленный" с алгебраической точки зрения итерационньй способ расширения множества обобщенных функций оказывается достаточно эффективным для введения требуемой топологии. На каждом шаге описанного процесса определяется своя топология, причем она оказьвается сильнейшей из всех, в смысле которых введенные на данный момент операции будут непрерьвными.

В качестве топологии на множестве $X_{1}=D_{p}$ выбираем образ топологии $D^{\prime}(\Omega)$ при действии оператора $I$. Тогда пространства $D_{p}$ и $D^{\prime}(\Omega)$ гомеоморфны. Для определения топологической структуры в классе натуральных распределений рассмотрим сначала множество $X_{2}$. Зададим на $\left(X_{1}\right)^{2}$ топологию произведения, а на $X_{2}$ финальную топологию относительно пары $\left(\left(X_{2}\right)^{2}, A_{1,1}\right)$. Введем топологию на $X_{r}, r \geqslant 2$, в предположении, что все топологические пространства $X_{m}$ при $m<r$ уже определены. В частности, задана топология на любых множествах $X_{m}$ и $X_{n}$ при $m+n=r$. Определим на $X_{m} \times X_{n}$ топологию произведения, а на $X_{r}$ финальную топологию относительно пары $\left(X_{m} \times X_{n}, A_{m, n}\right)$. Множество $X_{r}$ с этой топологией обозначим через $X_{r}^{m, n}$. Его топология является сильнейшей среди тех, для которых оператор $A_{m, n}$ оказьвается непрерьвньп [25]. Под топологическим пространством $X_{r}$ будем понимать данное множество с топологией, являющейся нижней гранью семейства топологий $\left\{X_{r}^{m, n}\right\}[25]$ по всем значениям $m$ и $n$, удовлетворяюшим равенству $m+n=r$. Тогда для любых $m$ и $n$ при $m+n=r$ оператор $A_{m, n}: X_{m} \times X_{n} \rightarrow X_{m+n}$ непрерьвен. Наконец, под топологическим пространством $D_{n}$ (или $D^{n}$, если под множествами $X_{m}$ и операторами $A_{m, n}$ понимаются соответствуюшие выражения из п. 1) будем понимать топологическую сумму семейства $\left\{X_{r}\right\}[25]$.

Топология в классе целых распределений определяется аналогично. В качестве топологии на $Y_{2}$ выбирается финальная топология относительно пары $\left(\left(Y_{2}\right)^{2}, B_{1,1}\right)$. Зададим топологию на множестве $Y_{r}, r \geqslant 2$, в предположении, что топологии $Y_{m}$ и $Y_{n}$ при $m+n=r$ определены. В качестве топологии $Y_{r}^{m, n}$ на $Y_{r}$ выбирается финальная топология в смысле пары $\left(Y_{m} \times Y_{n}, B_{m, n}\right)$. Под топологическим пространством $Y_{r}$ будем понимать это множество, наделенное топологией нижней грани семейства топологий $\left\{Y_{r}^{m, n}\right\}, m+n=r$. Тогда для любых $m, n$ таких, что $m+n=r$, оператор $B_{m, n}: Y_{m} \times Y_{n} \rightarrow Y_{r}$ является непрерывным. Зададим на $D_{i}$ топологию суммы семейства $\left\{Y_{r}\right\}$. Топологическое пространство $D^{i}$ вводится аналогично.

Установим некоторые свойства полученных топологических пространств.

Теорема 4. Операиия умножсния на $D_{n}$ непрерывна. 
ДокаЗАТЕЛьство. Для любых $x, y \in D_{n}$ существуют номера $m$ и $n$ такие, что справедливы включения $x \in X_{m}, y \in X_{n}$. Тогда их произведение принадлежит пространству $X_{r}$, где $m+n=r$, причем $x \cdot y=A_{m, n}(x, y)$. Для любой окрестности $U$ точки $x \cdot y$ в силу непрерывности оператора $A_{m, n}$ существует окрестность $V$ пары $(x, y)$ на $X_{m} \times X_{n}$ такая, что справедливо вложение $A_{m, n} V \subset U$. Пользуясь определением топологии произведения [25], установим равенство $V=V_{1} \times V_{2}$, где $V_{1}$ и $V_{2}-$ окрестности точек $x$ и $y$ соответственно в $X_{m}$ и $X_{n}$. Учитьвая непрерывность канонического вложения этих пространств в $D_{n}$, заключаем, что $V_{1}$ и $V_{2}$ являются окрестностями точек $x$ и $y$ в топологии $D_{n}$. Согласно определению операции умножения на $D_{n}$ справедливо равенство

$$
A_{m, n}\left(V_{1} \times V_{2}\right)=V_{1} \cdot V_{2}=\left\{\xi \cdot \eta \mid \xi \in V_{1}, \eta \in V_{2}\right\}
$$

Итак, для любой окрестности $U$ произведения $x \cdot y$ сушествуют окрестности $V_{1}$ и $V_{2}$ точек $x$ и $y$ такие, что имеет место вложение $V_{1} \cdot V_{2} \subset U$, откуда следует утверждение теоремы.

Теорема 5. Вложение $D_{p}$ в $D_{n}$ непрерывно, а $Y_{X}$ плотно.

ДокАЗАТЕЛЬСТво. Пространство $D_{n}$ является топологической суммой семейства $\left\{X_{r}\right\}$. Каноническое вложение $X_{1}$ и $D_{n}$ непрерьвно. Тогда непрерывность вложения $D_{p}$ в $D_{n}$ следует из равенства $X_{1}=D_{p}$.

Для любой точки $x \in D_{n}$ существует номер $m$ такой, что $x \in X_{m}$. Тогда найдутся простые распределения $x^{1}, \ldots, x^{m}$, образующие мультипликативное разложение $x$. Оператор $A:\left(D_{p}\right)^{m} \rightarrow X_{m}$, характеризуемьй равенством $A\left(x^{1}, \ldots, x^{m}\right)=x$, непрерьвен. Следовательно, для любой окрестности $U$ точки $x$ существуют окрестности $U_{1}, \ldots, U_{m}$ точек $x^{1}, \ldots, x^{m}$ соответственно такие, что $U_{1} \cdot \ldots \cdot U_{m} \subset U$.

Множество $D(\Omega)$ плотно в $D^{\prime}(\Omega)$, гомеоморфном $D_{p}$. Тогда вложение $Y_{X}=I(D(\Omega))$ в $D_{p}$ плотно. Следовательно, пересечение $U_{i} \cap Y_{X}$ для любого $i=1, \ldots, m$ непусто. В результате получаем соотношение

$$
\prod_{i=1}^{m} U_{i} \cap Y_{X} \neq \varnothing
$$

Тогда пересечение $\left(U_{1} \cdot \ldots \cdot U_{m}\right) \cap Y_{X}$ также непусто. Учитьвая вложение произведения множеств $\left\{U_{i}\right\}$ в $U$, заключаем, что $U \cap Y_{X} \neq \varnothing$. Таким образом, произвольная окрестность точки $x$ имеет непустое пересечение с множеством $Y_{X}$, т.е. является его точкой прикосновения. Отсюда в силу произвольности выбора точки $x \in D_{n}$ следуют утверждения теоремы.

ТЕОРема 6. Операчии сложения, умножсения и дифферениирования непрерывны на $D_{i}$.

ДоказАТЕЛьство. Непрерьвность сложения на $D_{i}$ доказьвается так же, как непрерьвность умножения на $D_{p}$. Для любых $x, y \in D_{i}$ существуют номера $m$ и $n$ такие, что $x \in Y_{m}, y \in Y_{n}$. Тогда найдутся натуральные распределения $x^{1}, \ldots, x^{m} ; y^{1}, \ldots, y^{n}$, 
суммы которых составляют $x$ и $y$. Пользуясь определением произведения на множестве $D_{i}$, устанавливаем равенство

$$
x \cdot y=\sum_{i=1}^{m} \sum_{j=1}^{n} x^{i} \cdot y^{j} .
$$

Таким образом, произведение на множестве $D_{i}$ сводится к сумме произведений натуральных распределений. Учитывая непрерьвность умножения на $D_{n}$ и сложения на $D_{i}$, заключаем, что операция умножения непрерывна на $D_{i}$. Непрерьвность дифференцирования на $D_{i}$ является следствием аналогичного свойства на $D_{p}$ и непрерьвности операций сложения и умножения. Теорема доказана.

По аналогии с теоремой 4 доказьвается следующее утверждение.

Теорема 7. Вложсение $D_{p}$ в $D_{i}$ непрерывно, а $Y_{X}$ плотно.

Естественно, топологическиепространства $D^{n}$ и $D^{i}$ обладают аналогичными свойствами. На основе полученных результатов приходим к заключению.

Теорема 8. Мнохсество иелых распределений $D^{i}$ (соответственно $D_{i}$ ) обладает структурой топологической дифференциальной коммутативной ассочиативной (соответственно неассоциативной) алгебры.

Итак, построены классы целых распределений, сушественно более широкие, чем классическоепространство распределений, замкнутые относительно операций сложения, умножения и дифференцирования, на которых введена топология, согласованная с алгебраической структурой множества. Полученные результаты могут быть использованы для анализа широкого класса нелинейных бесконечномерных систем. В частности, для многих важных с практической точки зрения задач математической физики нелинейные члены характеризуются операцией умножения (произведение неизвестных функций, функции на производную и т.п., см., например, [20]). Если для такой системы получены даже весьма слабые априорные оценки решения (лиш бы было непрерьвное вложение соответствующего функционального класса в пространство распределений), то, пользуясь непрерывностью операций на множествах арифметических распределений, можно обосновать предельньй переход и установить желаемьй результат. Получаемое решение оказывается не просто арифметическим распределением, но и принадлежит тому функциональному классу, на котором были получены априорные оценки решения, т.е. оказьвается вполне регулярньм объектом. Однако, даже доказательство разрешимости задачи на множествах арифметических распределений нельзя считать неудовлетворительным результатом, поскольку объекты такой природы могут быть сколь угодно точно аппроксимированы достаточно регулярными функциями. Вспомним также, что решения широкого класса дифференциальных уравнений могут быть интерпретированы как обобщенные распределения [14]-[19].

4. Бинарные отношения. Выше был предложен итерационньй процесс, позволяющий осуществить расширение множества обобщенных функций так, чтобы на нем были определены и непрерьвны естественные операции. При этом практически нигде не 
использовалась собственно природа распределений. В связи с этим возникает предположение, что описанную процедуру можно формализовать и применить для непрерьвного продолжения структур общего вида. Мы ограничимся рассмотрением лишњ бинарных отношений.

Рассмотрим отделимое топологическое пространство $X$ с топологией $\tau$ и подмножеством $Y$, на котором определено бинарное отношение $\rho$. Предположим, что множество $Y$ секвенциально плотно в $X$ в том смысле, что для любой точки $x$ из $X$ существует последовательность из $Y$, сходящаяся к $x$. Определим семейство $\Psi$ всех сходяшихся последовательностей из $Y$ и отношение эквивалентности $\psi$ на нем, считая условие $\left\{x_{k}\right\} \psi\left\{y_{k}\right\}$ выполненным, если соответствуюшие пределы совпадают. Рассмотрим фактормножество $X_{Y}=\Psi / \psi$. Для любого $x \in X$ определим класс эквивалентности $I x=\left[\left\{x_{k}\right\}\right]_{\psi}$ по отношению $\psi$ с представителем $\left\{x_{k}\right\}$ - последовательностью элементов из $Y$, сходящейся к $x$. Тем самым, задана биекция $I: X \rightarrow X_{Y}$, которую назовем секвенциальным изоморфизмом пространства $X$ относительно множества $Y$. Положим $Y_{X}=I(Y)$.

ОПРЕДЕЛЕНИЕ 1. Тройку $\left(X_{\rho}, \rho_{\tau}, \tau_{\rho}\right)$ назовем секвенииальным продолжением тройки $(Y, \rho, \tau)$, если вьполнены следующие условия:

1) $X_{Y}$ - подмножество $X_{\rho}$;

2) $\rho_{\tau}$ - оператор на $X_{\rho}$;

3) множество $\rho_{\tau}\left(Y_{X}\right)$ равномощно $\rho(Y)$;

4) $\tau_{\rho}$ - топология на $X_{\rho}$;

5) топология, индуцированная $\tau_{\rho}$ на $X_{Y}$, гомеоморфна $\tau$;

6) отображение $\rho_{\tau}$ непрерьвно в смысле $\tau_{\rho}$.

Положим $\Psi_{1}=\Psi, \psi_{1}=\psi, X_{1}=X_{Y}$. Пусть $\beta(Y)$ есть булеан множества $Y$. Определим оператор $\rho^{1}: Y \rightarrow \beta(Y)$, полагая $\rho^{1}(y)=\rho(y)$ для всех $y \in Y$, где $\rho(y)$ - образ отношения $\rho$ в точке $y$, являюшийся подмножеством $Y$, а значит, элементом $\beta(Y)$. Для любого множества $x$ из $Y$ семейство $\rho^{1}(x)$ является подмножеством $\beta(Y)$. В частности, для последовательности $\left\{x_{k}\right\}$ элементов множества можно определить выражение $\rho^{1}\left(\left\{x_{k}\right\}\right)=\rho\left(\left\{x_{k}\right\}\right)=\left\{\rho\left(x_{k}\right)\right\}$, являющееся подмножеством $\beta(Y)$.

Зададим оператор $\rho^{2}: \beta(Y) \rightarrow \beta^{2}(Y)$, где $\beta^{2}(Y)=\beta[\beta(Y)]$, полагая $\rho^{2}(x)=\rho^{1}(x)$ для всех $x \in \beta(Y)$. Имеем включение $\rho^{2}\left(\left\{x_{k}\right\}\right) \in \beta^{2}(Y)$. Учитьвая вложение $\Psi_{1} \subset$ $\beta(Y)$, устанавливаем, что $\rho^{2}\left(\Psi_{1}\right) \subset \beta^{2}(Y)$. Определим семейство

$$
\Psi_{2}=\rho^{2}\left(\Psi_{1}\right)=\left\{\rho^{2}\left(\left\{x_{k}\right\}\right) \mid\left\{x_{k}\right\} \in \Psi_{1}\right\}=\left\{\left\{\rho\left(x_{k}\right)\right\} \mid\left\{x_{k}\right\} \in \Psi_{1}\right\} .
$$

Введем на нем эквивалентность $\psi_{2}$, считая условие $x \psi_{2} x^{\prime}$ выполненным, если существуют элементы $y \in\left(\rho^{2}\right)^{-1}(x), y^{\prime} \in\left(\rho^{2}\right)^{-1}\left(x^{\prime}\right)$ такие, что справедливо соотношение $y \psi_{1} y^{\prime}$. Зададим фактормножество $X_{2}=\Psi_{2} / \psi_{2}$ и оператор $\rho_{1}: X_{1} \rightarrow X_{2}$ следуюшим образом:

$$
\rho_{1}(x)=\left[\rho^{2}(y)\right]_{2} \quad \forall y \in x \in X_{1}
$$

Продолжая описанньй процесс, для произвольного номера $m$ введем оператор $\rho^{m}$ : $\beta^{m-1}(Y) \rightarrow \beta^{m}(Y)$, полагая $\rho^{m}(x)=\rho^{m-1}(x)$ для всех $x \in \beta^{m-1}(Y)$. Учитьвая вложение $\Psi_{m-1} \subset \beta^{m-1}(Y)$, устанавливаем, что $\rho^{m}\left(\Psi_{m-1}\right) \subset \beta^{m-1}(Y)$. Рассмотрим семейство

$$
\Psi_{m}=\rho^{m-1}\left(\Psi_{m-1}\right)=\left\{\rho^{m-1}(x) \mid x \in \Psi_{m-1}\right\}
$$


и отношение эквивалентности $\psi_{m}$ на нем, считая условие $x \psi_{m} x^{\prime}$ вьполненным, если существуют элементы $y \in\left(\rho^{m}\right)^{-1}(x), y^{\prime} \in\left(\rho^{m}\right)^{-1}\left(x^{\prime}\right)$ такие, что справедливо соотношение $y \psi_{m-1} y^{\prime}$. Определим фактормножество $X_{m}=\Psi_{m} / \psi_{m}$ и оператор $\rho_{m-1}$ : $X_{m-1} \rightarrow X_{m}$ из условия

$$
\rho_{m-1}(x)=\left[\rho^{m}(y)\right]_{m} \quad \forall y \in x \in X_{m-1}
$$

Рассмотрим объединение $X_{\rho}$ семейства $\left\{X_{m}\right\}$. Для любого элемента $x$ из $X_{\rho}$ найдется номер $m$ такой, что справедливо включение $x \in X_{m}$. Определим оператор $\rho_{\tau}: X_{\rho} \rightarrow$ $X_{\rho}$, полагая $\rho_{\tau}(x)=\rho_{m}(x)$ для любого $x \in X_{m}$ и произвольного $m$. Итак, построены расширение $X_{\rho}$ множества $X_{Y}$ и оператор $\rho_{\tau}$ на $X_{\rho}$. Тем самьм, вьполняются условия 1), 2) определения 1 .

Для любого элемента $y$ из образа $\rho(Y)$ сушествует элемент $x \in Y$ такой, что $y=$ $\rho(x)$. Тогда выражение

$$
\bar{y}=\rho_{\tau}(I x)=[\{\rho(x)\}]_{2},
$$

где $\{\rho(x)\}$ - стационарная последовательность с элементом $\rho(x)$, принадлежит множеству $\rho_{\tau}\left(Y_{X}\right)$. Предположим, что существует объект $x^{\prime}$, отличньй от $x$, такой, что $y=$ $\rho\left(x^{\prime}\right)$. Справедливы равенства

$$
\bar{y}^{\prime}=\rho_{\tau}\left(I x^{\prime}\right)=\left[\left\{\rho\left(x^{\prime}\right)\right\}\right]_{2}=[\{\rho(x)\}]_{2}=\bar{y},
$$

т.е. соответствуюшие элементы множества $\rho_{X}\left(Y_{X}\right)$ совпадают. Тогда любой объект из $\rho(Y)$ однозначно определяет элемент множества $\rho_{\tau}\left(Y_{X}\right)$.

$\mathrm{C}$ другой стороны, для любого $\bar{y} \in \rho_{\tau}\left(Y_{X}\right)$ найдется элемент $\bar{x} \in Y_{X}$ такой, что $\bar{y}=\rho(\bar{x})$. Определяя $x=I^{-1} \bar{x}$, найдем $y=\rho(x)$ из множества $\rho(Y)$. Пусть существует другой элемент $\bar{x}^{\prime} \in Y_{X}$, удовлетворяющий равенству $\bar{y}=\sigma\left(\bar{x}^{\prime}\right)$. Полагая $y^{\prime}=\rho\left(x^{\prime}\right)$, где $x^{\prime}=I^{-1} \bar{x}^{\prime}$, устанавливаем соотношения

$$
\rho_{\tau}(\bar{x})=[\{\rho(x)\}]_{2}=[\{y\}]_{2}=\rho_{\tau}\left(\bar{x}^{\prime}\right)=\left[\left\{\rho\left(x^{\prime}\right)\right\}\right]_{2}=[\{y\}]_{2} .
$$

Таким образом, стационарные последовательности $\{y\}$ и $\left\{y^{\prime}\right\}$ эквивалентны в смысле отношения $\psi_{2}$, что возможно исключительно при $y=y^{\prime}$. Итак, любой элемент из $\rho_{X}\left(Y_{X}\right)$ однозначно определяет элемент множества $\rho(Y)$. Следовательно, множества $\rho(Y)$ и $\rho_{\tau}\left(Y_{X}\right)$ равномошны.

Введем топологию на множестве $X_{\rho}$. Обозначим через $\tau_{1}$ топологию на $X_{1}$, перенесенную из $X$ с помощю биекции $I$, т.е. $\tau_{1}=I \tau$. Тогда пространства $\left(X_{1}, \tau_{1}\right)$ и $(X, \tau)$ гомеоморфны. Обозначим через $\tau_{2}$ финальную топологию на $X_{2}$, перенесенную из $\left(X_{1}, \tau_{1}\right)$ с помощью оператора $\rho_{1}$. Тогда отображение $\rho_{1}:\left(X_{1}, \tau_{1}\right) \rightarrow\left(X_{2}, \tau_{2}\right)$ непрерывно. Для произвольного номера $m$, обозначая через $\tau_{m}$ финальную топологию на $X_{m}$, перенесенную из $\left(X_{m-1}, \tau_{m-1}\right)$ с помощью оператора $\rho_{m-1}$, установим непрерьвность отображения $\rho_{m-1}:\left(X_{m-1}, \tau_{m-1}\right) \rightarrow\left(X_{m}, \tau_{m}\right)$. Пусть $\tau_{\rho}$ есть топология на $X_{\rho}$, соответствуюшая топологической сумме пространств $\left\{\left(X_{m}, \tau_{m}\right)\right\}$. Тогда $\rho_{\tau}$ - непрерьвньй оператор на $\left(X_{\rho}, \tau_{\rho}\right)$. При этом топология, индуцированная $\tau_{\rho}$ на $X_{Y}$, есть $\tau_{1}$, которая гомеоморфна $\tau$. Итак, выполняются свойства 4)-6) определения 1. Таким образом, доказано утверждение. 
ТЕорема 9. Тройка $\left(X_{\rho}, \rho_{\tau}, \tau_{\rho}\right)$ - секвенциальное продолжение $(X, \rho, \tau)$.

Установим дополнительньй результат.

Tеорема 10. Множество $\Sigma_{x}=\bigcup_{m}\left(\rho_{\tau}\right)^{m}\left(Y_{X}\right)$ плотно в $X_{\rho}$.

ДокАЗАТЕЛЬСтво. Поскольку оператор $I: X \rightarrow X_{Y}$ - гомеоморфизм, а $Y$ плотно в $X$, то множество $Y_{X}$ плотно в $X_{Y}=X_{1}$, а значит, справедливо равенство $\bar{Y}_{X}=X_{1}$. В силу непрерывности $\rho_{1}$ имеем вложение

$$
\rho_{1}\left(\bar{Y}_{X}\right) \subset \overline{\rho_{1}\left(Y_{X}\right)}
$$

Оператор $\rho_{1}$ является сюръекцией, т.е. $\rho_{1}\left(X_{1}\right)=X_{2}$. В результате получаем $X_{2} \subset$ $\overline{\rho_{1}\left(Y_{X}\right)}$. Откуда следует, что $X_{2}=\overline{\rho_{1}\left(Y_{X}\right)}$, т.е. множество $\rho_{1}\left(Y_{X}\right)$ плотно в $X_{2}$. Учитывая, что сужение оператора $\rho_{\tau}$ на $X_{1}$ совпадает с $\rho_{1}$, заключаем, что $\rho_{\tau}\left(Y_{X}\right)$ плотно в $X_{2}$. Пусть множество $\rho_{\tau}^{m-1}\left(Y_{X}\right)$ плотно в $X_{m}$. Оператор $\rho_{1}: X_{m} \rightarrow X_{m+1}$ - непрерьвная сюръекция. Тогда

$$
X_{m+1}=\rho_{m}\left(X_{m}\right) \subset \rho_{m}\left(\overline{\rho_{\tau}^{m-1}\left(Y_{\tau}\right)}\right) \subset \overline{\rho_{m}\left(\rho_{\tau}^{m-1}\left(Y_{X}\right)\right)} \subset X_{m+1} .
$$

Отсюда следует плотность вложения в $X_{m+1}$ множества

$$
\rho_{\tau}^{m}\left(Y_{X}\right)=\rho_{m}\left(\rho_{\tau}^{m-1}\left(Y_{X}\right)\right)
$$

Пусть $U$ есть непустое открытое множество из пространства $X_{\rho}$. Тогда существует номер $m$ такой, что имеет место включение $U \in \tau_{m}$. В силу плотности вложения множества $\rho_{\tau}^{m-1}\left(Y_{X}\right)$ в $X_{m}$ имеем $\rho_{\tau}^{m-1}\left(Y_{X}\right) \cap U \neq \varnothing$, откуда следует неравенство $\Sigma_{X} \cap U \neq \varnothing$. Учитывая произвольность окрестности $U$, устанавливаем, что $\Sigma_{X}$ плотно в $X_{\rho}$. Теорема доказана.

Если множество $Y$ не секвенциально плотно, а только плотно в топологическом пространстве $X$, то для любой точки $x$ из $X$ существует фильтр $F$ из $Y$, сходяшийся к $x$. Обозначим через $\Psi$ множество всех сходящихся фильтров из $Y$. Определим на нем отношение эквивалентности $\psi$, считая условие $F \psi F^{\prime}$ выполненньп, если указанные фильтры имеют один и тот же предел. При этом фактормножество $X_{Y}=\Psi / \psi$ равномошно $X$, т.е. существует биекция $I: X \rightarrow X_{Y}$. Теперь можно повторить приведенные ранее рассуждения и построить секвенциальное продолжение бинарного отношения при соответствующей корректировке технических выкладок.

В качестве приложения полученных результатов определим секвенциальное продолжение операции первого порядка. Рассмотрим отделимое топологическоепространство $(X, \tau)$ и плотное подмножество $Y$. Пусть множества $\Psi, X_{Y}, Y_{X}$, отношение $\psi$ и оператор $I$ определяются указанным вьше способом. Предположим, что на $Y$ задана операция первого порядка, характеризуемая оператором $A: Y \rightarrow Y$. Будем считать, что топологическая и алгебраическая структура на $Y$ согласованы, т.е. оператор $A$ непрерывен в топологии, индуцированной на $Y$ топологией $\tau$. График этого оператора является бинарным отношением на $Y$. Поэтому для продолжения операции можно применить описанную методику. При этом, пользуясь свойствами рассматриваемого отношения, можно существенно упростить проводимые рассуждения. 
ОПРЕДЕЛЕНИЕ 2. Тройку $\left(X_{A}, A_{\tau}, \tau_{A}\right)$ назовем секвенииальным продолжением тройки $(Y, A, \tau)$, если вьполнены условия:

1) $X_{Y}$ - подмножество $X_{A}$;

2) $A_{\tau}$ - оператор на $X_{A}$;

3) $A_{\tau}\left(Y_{X}\right)$ равномощно $A(Y)$;

4) $\tau_{A}$ - топология на $X_{A}$;

5) топология, индуцированная $\tau_{A}$ на $X_{Y}$, гомеоморфна $\tau$;

6) $A_{\tau}$ непрерьвен в смысле $\tau_{A}$.

Положим $\Psi_{1}=\Psi, \psi_{1}=\psi$. Определим семейство $\Psi_{2}=A\left(\Psi_{1}\right)$ и эквивалентность $\psi_{2}$ на нем, считая условие $M \psi_{2} M^{\prime}$ вьполненным, если сушествуют фильтры $F, F^{\prime}$ из $\Psi_{1}$ такие, что имеют место соотношения $M=A(F), M^{\prime}=A\left(F^{\prime}\right), F \psi_{1} F^{\prime}$. Для любого номера $m$ рассмотрим семейство $\Psi_{m+1}=A\left(\Psi_{m}\right)$ и эквивалентность $\psi_{m+1}$ на нем, причем условие $M \psi_{m+1} M^{\prime}$ предполагает существование величин $F, F^{\prime}$ из $\Psi_{m}$ таких, что выполняются соотношения $M=A(F), M^{\prime}=A\left(F^{\prime}\right), F \psi_{m} F^{\prime}$. Определим фактормножества $X_{m}=\Psi_{m} / \psi_{m}, m=1,2, \ldots$, и оператор $A_{m}: X_{m} \rightarrow X_{m+1}$ с помошью равенства

$$
A_{m} x=[\{A(F)\}]_{m+1} \quad \forall x=[\{F\}]_{m} \in X_{m} .
$$

Обозначим через $X_{A}$ объединение семейства $\left\{X_{m}\right\}$. Для любого элемента $x$ из $X_{A}$ существует номер $m$ такой, что $x \in X_{m}$. На множестве $X_{A}$ определим оператор $A_{\tau}$, полагая $A_{\tau} x=A_{m} x$ для всех $x \in X_{m}$.

Для любого $y \in A(Y)$ сушествует элемент $x$ из $Y$ такой, что имеет место равенство $y=A x$. Определим значение

$$
\bar{y}=A_{\tau}(I x)=A_{1}(I x)=[A(F)]_{2},
$$

где $F$ - произвольный фильтр из $Y$, сходяшийся к точке $x$. Учитывая непрерывность оператора $A$, заключаем, что $A(F)$ есть фильтр из $Y$, сходяшийся к $A x$. Таким образом, значение $\bar{y} \in A_{\tau}\left(Y_{X}\right)$ однозначно определяется элементом $y$. С другой стороны, для любого элемента $\bar{y} \in A_{\tau}\left(Y_{X}\right)$ сушествует значение $x \in Y_{X}$ такое, что $\bar{y}=A_{\tau} \bar{x}$. Тогда можно определить точку $x=I^{-1}(\bar{x})$ и $y=A x$ из множества $Y$. Предположим, что найдется другое значение $x^{\prime} \in Y_{X}$, удовлетворяющее условию $\bar{y}=A_{\tau} \bar{x}^{\prime}$. Ему соответствует $y^{\prime}=A x^{\prime}$, где $x^{\prime}=I^{-1}\left(\bar{x}^{\prime}\right)$. При этом выполняются соотношения

$$
A_{\tau}(\bar{x})=[A(F)]_{2}=A_{\tau}\left(\bar{x}^{\prime}\right)=\left[A\left(F^{\prime}\right)\right]_{2},
$$

где $F, F^{\prime}$ - произвольные фильтры, сходяшиеся соответственно к точкам $x, x^{\prime}$. В качестве таковых выбираем элементарные фильтры, ассоциированные со стационарньми последовательностями, определяемыми этими точками [25]. Последние равенства возможны исключительно при вьполнении условия $A x=A x^{\prime}$, а значит, $y=y^{\prime}$. Таким образом, между множествами $A_{\tau}\left(Y_{X}\right)$ и $A(Y)$ можно установить взаимно однозначное соответствие, т.е. они равномощны.

Определив на $X_{2}$ образ относительно оператора $A_{1}$ топологии из $X_{1}$, получаем сильнейшую топологию, относительно которой оператор $A_{1}: X_{1} \rightarrow X_{2}$ непрерьвен. Аналогично на множестве $X_{m+1}$ вводится образ относительно $A_{m}$ топологии $X_{m}$. Тогда оператор $A_{m}$ непрерьвен. Понимая под топологическим пространством $X_{A}$ топологическую сумму семейства $\left\{X_{m}\right\}$, устанавливаем, что оператор $A_{\tau}$ непрерывен в топологии $X_{A}$. Таким образом, справедливо утверждение. 
Теорема 11. Тройка $\left(X_{A}, A_{\tau}, \tau_{A}\right)$ - секвенииальное продолжсене $(Y, A, \tau)$.

Аналог теоремы 10 в данном случае принимает достаточно простой вид.

Tеорема 12. Мнохество $Y_{X}$ плотно в $X_{A}$.

ДоКАЗАТЕЛЬСТВо. Для любой точки $x$ из $Y_{X}$ справедливо равенство

$$
A_{\tau}(x)=[A(F)]_{\psi}
$$

где $F$ - произвольный фильтр, сходяшийся к точке $I^{-1} x$. Тогда $A(F)$ является фильтром на $Y$, сходяшимся к элементу $A\left(I^{-1} x\right)$, а значит, принадлежит классу $\Psi$. Справедливо включение $A_{\tau}(x) \in X_{Y}$. Найдем значение

$$
I^{-1}\left(A_{\tau}(x)\right)=I^{-1}\left([A(F)]_{\psi}\right)=\lim A(F)=A\left(I^{-1} x\right)
$$

Таким образом, $I^{-1}\left(A_{\tau}(x)\right) \in Y$, откуда следует, что $A_{\tau}(x) \in Y_{X}$ для любого элемента $x$ из $Y_{X}$. Значит, $A_{\tau}$ есть оператор на $Y_{X}$.

В силу плотности вложения множества $Y$ в $X$ вложение $Y_{X}$ плотно в $X_{1}$. Пусть $Y_{X}$ плотно вложено в пространство $X_{m}$. Для любой точки $x$ из $X_{m+1}$ существует $y$ из $X_{m}$, удовлетворяюший равенству $x=A_{m} y$. Учитьвая непрерывность оператора $A_{m}$, устанавливаем, что для любой окрестности $U$ точки $x$ существует окрестность $V$ точки $y$ такая, что имеет место вложение $A_{m}(V) \subset U$. В силу плотности вложения множества $Y_{X}$ в $X_{m}$ справедливо соотношение $V \cap Y_{X} \neq \varnothing$, а значит, сушествует некоторая точка $z \in V \cap Y_{X}$. Из условия $z \in V$ следует, что $A_{m}(z) \in A_{m}(V)$. С другой стороны, из включения $z \in Y_{X}$, поскольку $A_{\tau}$ является оператором на $Y_{X}$, получаем, что $A_{m}(z) \in Y_{X}$. Таким образом, $A_{m}(z) \in A_{m}(V) \cap Y_{X}$, и множество $A_{m}(V) \cap Y_{X}$ непусто. Отсюда следует соотношение $U \cap Y_{X} \neq \varnothing$. Учитывая произвольность окрестности $U$ и точки $x$, заключаем, что вложение $Y_{X}$ в $X_{m+1}$ плотно. Таким образом, множество $Y_{X}$ плотно вложено в любое из пространств $X_{m}$, а значит, и в $X_{A}$. Теорема доказана.

Очевидно, любая структура на множестве $X$ может быть перенесена на $X_{Y}$ с помощью биекции $I$ [26]. В связи с этим можно отождествить эти множества и считать, что оператор $A_{\tau}$ (точнее, его сужение $A_{1}$ ) является (с точностью до изоморфизма) непрерьвньп продолжением оператора $A$ на $X$.

В качестве приложения определим дифференцирование распределений. На множестве $D(\Omega)$ рассмотрим дифференциальный оператор $\partial$ произвольного порядка. Обозначим через $\partial^{\prime}$ оператор обобщенного дифференцирования, соответствующий $\partial$, а через $I$ секвенциальньй изоморфизм $D^{\prime}(\Omega)$ относительно $D(\Omega)$.

ТЕорема 13. Тройка $\left(I\left(D^{\prime}(\Omega)\right), I \partial^{\prime} I^{-1}, I \tau\right)$ является секвенциальным продолжением тройки $(D(\Omega), \partial, \tau)$.

ДокАЗАтЕльство. Определим, как и раньше, множество $\Psi$ и эквивалентность $\psi$ на нем. Введем обозначения $\Psi_{1}=\Psi, \psi_{1}=\psi, X_{1}=\Psi_{1} / \psi_{1}$. Для произвольного номера $m$ определим семейство

$$
\Psi_{m+1}=\left\{\left\{y_{k}\right\} \subset D(\Omega) \mid \exists\left\{x_{k}\right\} \in \Psi_{m}: y_{k}=\partial x_{k} \quad \forall k\right\}
$$


Условие $\left\{y_{k}\right\} \psi_{m+1}\left\{y_{k}^{\prime}\right\}$ на нем предполагает существование последовательностей $\left\{x_{k}\right\}$, $\left\{x_{k}^{\prime}\right\}$ таких, что вьполняются соотношения

$$
\left\{x_{k}\right\} \psi_{m}\left\{x_{k}^{\prime}\right\}, \quad y_{k}=\partial x_{k}, \quad y_{k}^{\prime}=\partial x_{k}^{\prime} \quad \forall k
$$

Положим $X_{m+1}=\Psi_{m+1} / \psi_{m+1}$. Пусть $X_{D}$ есть объединение семейства $\left\{X_{m}\right\}$.

Для любой последовательности $\left\{y_{k}\right\} \in \Psi_{2}$ существует последовательность $\left\{x_{k}\right\} \in$ $\Psi_{1}$, сходящаяся к некоторой точке $x$, такая, что $y_{k}=\partial x_{k}$ для всех $k$. В силу непрерывности обобщенного дифференцирования имеет место сходимость $\partial^{\prime} x_{k} \rightarrow \partial^{\prime} x$ в $D^{\prime}(\Omega)$. Учитьвая совпадение классического и обобщенного дифференцирования на $D(\Omega)$, получаем $y_{k} \rightarrow \partial^{\prime} x$ в $D^{\prime}(\Omega)$. Тогда справедливо включение $\left\{x_{k}\right\} \in \Psi$, а значит, $\Psi_{2} \subset \Psi_{1}$. Аналогичным образом для любого номера $m$ устанавливаем вложение $\Psi_{m+1} \subset \Psi_{m}$.

При $\left\{y_{k}\right\} \psi_{2}\left\{y_{k}^{\prime}\right\}$ на $\Psi_{2}$ существуют последовательности $\left\{x_{k}\right\},\left\{x_{k}^{\prime}\right\}$ из $D(\Omega)$, сходяшиеся к одной и той же точке $x \in D^{\prime}(\Omega)$, такие, что

$$
y_{k}=\partial x_{k}, \quad y_{k}^{\prime}=\partial x_{k}^{\prime} \quad \forall k
$$

Учитьвая непрерывность обобшенного дифференцирования, получаем сходимость $y_{k} \rightarrow \partial^{\prime} x$ и $y_{k}^{\prime} \rightarrow \partial^{\prime} x$ в $D^{\prime}(\Omega)$, откуда следует $\left\{y_{k}\right\} \psi_{1}\left\{y_{k}^{\prime}\right\}$. Аналогично из условия $\left\{y_{k}\right\} \psi_{m+1}\left\{y_{k}^{\prime}\right\}$ на $\Psi_{m+1}$ следует $\left\{y_{k}\right\} \psi_{m}\left\{y_{k}^{\prime}\right\}$ для любого $m$. Тогда $X_{m+1} \subset X_{m}$ и множество $X_{D}$ совпадает с $X_{1}$, т.е. с $I\left(D^{\prime}(\Omega)\right)$.

Найдем значение

$$
\partial_{\tau} x=\left[\left\{\partial x_{k}\right\}\right]_{\psi_{m}} \quad \forall\left\{x_{k}\right\} \in x \in X_{1}
$$

Для любой последовательности $\left\{x_{k}\right\}$ из класса $x \in X_{1}$ имеем сходимость $x_{k} \rightarrow I^{-1} x$ в $D^{\prime}(\Omega)$, а значит, $\partial x_{k} \rightarrow \partial^{\prime} I^{-1} x$ в $D^{\prime}(\Omega)$. Отсюда следует, что $\partial_{\tau}=I \partial^{\prime} I^{-1}$. В силу равенства $X_{D}=I\left(D^{\prime}(\Omega)\right)$ топология на нем совпадает с образом в смысле $I$ топологии на $D^{\prime}(\Omega)$, т.е. с $I \tau$. Теорема доказана.

Итак, секвенциальное продолжение операции дифференцирования с $D(\Omega)$ на $D^{\prime}(\Omega)$ с точностью до изоморфизма совпадает с обобщенным дифференцированием распределений. Полученные результаты показывают, что дифференпирование обобщенных функций может быть получено без перехода к сопряженному пространству. Это достаточно важно, поскольку известные обобшенные распределения, как правило, не могут быть интерпретированы как линейные непрерывные функционалы над каким-либо содержательным функциональньц классом (см., например, [15]-[17], а также определенные выше арифметические распределения). На основании проведенного анализа можно заключить, что дифференцирование как на множестве распределений, так и на его расширениях можно определить чисто алгебраическими средствами.

Нетрудно убедиться, что приведенньй ранее несогласованньй вариант множества арифметических распределений соответствует секвенциальному продолжению операций второго порядка, которое вводится по аналогии с определением 2. Имеется возможность формализовать и согласованный вариант определения класса арифметических распределений, но естественно с усложнением описанной процедуры. 


\section{СПИСОК ЦИТИРОВАННОЙ ЛИТЕРАТУРЫ}

[1] Скорняков Л. А. (ред. ) Общая алгебра. Т. 1, 2. М.: Наука, 1990, 1991.

[2] Крейн С. Г. (ред. ) Функциональный анализ. М.: Наука, 1972.

[3] Арнаутов В. И., Водинчар М. И., Михалёв А. В. Введениев теориютопологических колец и модулей. Кишинев, 1981.

[4] Dirac P. A. Theory of emission and absorption of radiation // Proc. Roy. Soc. Edinburgh. Sect. A. 1927. V. 114. P. 243-262.

[5] Schwartz L. Theorie des distributions. V. 1, 2. Paris: Hermann, 1950.

[5] Соболев С. Л. Некоторые применения функционального анализа в математической физике. Новосибирск: Изд. СО АН СССР, 1962.

[7] Владимиров В. С. Обобщенные функции в математической физике. М.: Наука, 1976.

[8] Антосик П., Микусинский Я., Сикорский Р. Теория обобщенных функций. Секвенциальный подход. М.: Мир, 1976.

[9] Tillmann H. G. Darstellung der shwartzchen distriburionen durch analytische funktionen // Math. Z. 1961. V. 77. № 2. P. 106-124.

[10] Бремерман Г. Распределения, комплексные переменные и преобразования Фурье. М.: Мир, 1968.

[11] Иванов В.К. Гиперраспределения и умножения распределений Шварца // Докл. АН CCCP. 1972. T. 204. № 5. C. 1045-1048.

[12] Keller K. On the Multiplication of Distribution. I. Preprint. Aachen: Techn. Hochschule, 1975.

[13] Berg L. Representations for distributions algebras // Z. Angew. Math. Mech. 1976. V. 56. № 5. P. 177-181.

[14] Маслов В. П. Распространение ударных волн в изоэнтропическом газе // Итоги науки и техн. Т. 8. М.: ВИНИТИ, 1977. С. 199-271.

[15] Colombeau J. F. A multiplication of distributions // J. Math. Anal. Appl. 1983. V. 94. № 1. P. 96-115.

[16] Colombeau J. F. New generalised functions and multiplication of distributions. Amsterdam: North-Holland, 1984.

[17] Егоров Ю. В. К теории обобщенных функций // УМН. 1990. Т. 45. № 5. С. 3-40.

[18] Шелкович В. М. Одна алгебра распределений и обобщенные решения нелинейных уравнений // Докл. АН СССР. 1995. Т. 342. № 5. С. 600-602.

[19] Rosinger E. E. Generalized solutions of nonlinear partial differential equations. AmsterdamOxford-New York: North-Holland, 1987.

[20] Лионс ЖК.-Л. Некоторые методы решения нелинейных краевых задач. М.: Мир, 1972.

[21] Гаевский Х., Грегер К., Захариас К. Нелинейные операторные уравнения и операторные дифференциальные уравнения. М.: Мир, 1978.

[22] Серовайский С.Я. Введение в арифметическую теорию распределений // Докл. АН Республики Казахстан. 1996. № 5. С. 7-13.

[23] Серовайский С.Я. Арифметическая теория распределений и одна нерешенная задача Ж.-Л. Лионса // Изв. АН Республики Казахстан. Сер. физ. -матем. 1996. № 3. С. 38-44.

[24] Schwartz L. Sur l'imposibilite de la multiplication des distributions // C. R. Acad. Sci. Paris. 1954. V. 239. № 15. P. 847-848.

[25] Бурбаки Н. Общая топология. Основные структуры. М.: Наука, 1968.

[26] Бурбаки Н. Теория множеств. М.: Мир, 1965.

Казахский государственный национальный университет им. аль-Фараби

Поступило 23.07 .96

Исправленный вариант 29.07.97 